Flores Tena, M.J., Ortega Navas, M.C. \& Sánchez Fuster, M.C (2021). Las nuevas tecnologías como estrategias innovadoras de enseñanza-aprendizaje en la era digital. Revista Electrónica Interuniversitaria de Formación del Profesorado, 24(1), 29-42.

DOI: https://doi.org/10.6018/reifop.406051

\title{
Las nuevas tecnologías como estrategias innovadoras de enseñanza-aprendizaje en la era digital
}

\author{
M. ${ }^{a}$ José Flores Tena ${ }^{1}, M .{ }^{a}$ del Carmen Ortega Navas ${ }^{2}, M^{a}$ Carmen Sánchez Fuster ${ }^{3}$ \\ ${ }^{1}$ Universidad Autónoma de Madrid, ${ }^{2}$ Universidad Nacional de Educación a Distancia (UNED) \\ ${ }^{3}$ Universidad de Murcia
}

\section{Resumen}

En el presente artículo nos preguntamos cuál es el papel de las nuevas tecnologías en las aulas y, más en concreto, en el proceso de enseñanza-aprendizaje de los futuros profesores. Además, nos planteamos si la enseñanza a distancia es adecuada para el buen desarrollo de dicho proceso, así como si para el desarrollo del conocimiento del estudiante y las nuevas tecnologías como herramienta docente son adecuadas al paradigma educativo actual, como estrategia de innovación educativa. Para ello se ha llevado a cabo un estudio de carácter cuantitativo, aplicando a los participantes, profesores de la Facultad de Educación de la Universidad Autonóma de Madrid que imparten su docencia en el Máster de Formación de Profesorado durante el curso 2018. Se trata de un cuestionario estructurado creado ad hoc. Las respuestas al cuestionario nos ha aportado datos sobre las necesidades e inquetudes que tiene el profesorado que forma a futuros profesorado de Educación Secundaria, respecto al uso de herramientas tecnológicas en el aula y su implicación como estrategia innovadora en el proceso de enseñanza-aprendizaje. El análisis de los resultados ha permitido comprobar que las las nuevas tecnologías constituyen un gran reto en las aulas en nuestros días, introduciendo nuevas metodologías de enseñanza que se pueden compaginar con la enseñanza tradicional.

\section{Palabras clave}

TIC; estrategias didácticas; enseñanza-aprendizaje; innovación y profesorado universitario.

\section{Contacto:}

María José Flores Tena, maria.flores@uam.es, Universidad Autónoma de Madrid, C/ Francisco Tomás y Valiente, 3. 28049 Madrid.

El texto presentado se vincula al Proyecto de Innovación Educativa "Estrategias didácticas y recursos audiovisuales eficaces en el TFM del Máster en Intervención Educativa en Contextos Sociales" (IP: Francisco. Javier García-Castilla). Ha sido elaborado por el Grupo de Innovación Docente EDIT (GID 2017-9) y financiado por la Universidad Nacional de Educación a Distancia (BICl 12/03/2018). 


\title{
New technologies as innovative teaching-learning strategies in the Digital Era
}

\begin{abstract}
New technologies are present in our daily lives and education is not exempt from them. In this article we ask ourselves what their role is in the classrooms and, more specifically, in the teaching-learning process. In addition, we ask whether distance education is adequate for the proper development of said process and for the development of student knowledge; as well as if new technologies as a teaching tool are appropriate to the educational paradigm. The global teaching-learning process is limited to the precise moment in which there is an effective transmission of information that originates from a sender (teachers) and reaches a receiver (students). The characteristic communicative act of the teaching-learning pedagogical process allows us to question ourselves about the suitability, the didactic adequacy and the impact of the use of new communication and information technologies in the classroom at the individual and social level. Besides, the strengths and weaknesses of learning about the use of new technologies in the classroom are analyzed.
\end{abstract}

\section{Key words}

ICT; innovative teaching-learning strategies; innovation and university faculty.

\section{Introducción}

Cabe destacar que los cambios que se han venido produciendo tras la irrupción de las Tecnologías de la Información y la Comunicación (TIC) digitales, caracterizadas por su simultaneidad, instantaneidad, interactividad, asincronía y ubicuidad, han abierto un nuevo abanico en las oportunidades y demandas en la educación. No obstante, el debate académico actual ha buscado centrarse, casi exclusivamente, en aspectos relacionados con la implementación, optimización y evaluación del uso de las tecnologías en el aula.

Las demandas de la sociedad han implicado que los docentes tengan que estar constantemente adaptándose a la integración de las TIC en el desempeño de su ejercicio profesional, y en consecuencia, en el conocimiento permanente para el proceso de formación de sus estudiantes. Del mismo modo, el desarrollo del aprendizaje permite al estudiante aprender a conocer y a ampliar la construcción de la enseñanza-aprendizaje.

Las nuevas generaciones tienen que aprender no solo a manejar grandes cantidades de información e interaccionar con un mundo tecnológico cambiante, sino también a desarrollar un concepto de sí mismo como ciudadano del mundo al mismo tiempo de conservar su identidad local (Bruner, 2013, p. 181).

La enseñanza en la escuela se caracteriza por el cambio pedagógico, entendido como un acto comunicativo, que permite ahondar en la adecuación didáctica, la idoneidad y el verdadero impacto del uso de las nuevas tecnologías de la comunicación y de la información en el aula. De hecho, el proceso integral de enseñanza-aprendizaje puede circunscribirse al instante preciso en el que se produce una transmisión efectiva de información. No debemos de perder de vista que el uso de las TIC por los estudiantes es cada vez más incentivado por los docentes para que aquéllos presenten sus tareas en formatos tecnológicos, innovadores y modernos. 
Es importante brindarles material para que lean en línea, ya sea publicando en el blog del curso o derivándolos a sitios Web que ofrezcan material relevante y confiable para la asignatura a partir de los cuales se puedan llevar a cabo actividades de aprendizaje (Pellizzon, 2012, p. 35).

Las condiciones en que se han venido transmitiendo la información en las últimas décadas, está llevando a extremos inimaginables con relación a las estructuras de la comunicación misma con respecto a otros tiempos. En tal sentido, la irrupción de las nuevas tecnologías de la información y la comunicación digitales han venido transformando radicalmente las formas actuales en que se producen, gran parte, de las relaciones sociales interpersonales docentes-discentes en el aula y fuera de ella.

Así, la dinámica de la revolución tecnológica ha supuesto la irrupción de los medios digitales, las TIC que están modificando las relaciones sociales y desmontado el andamiaje conceptual de los elementos presentes en la comunicación (Miralles, Gómez, Arias y Fontal, 2019). En efecto, Dallas W. Smythe el reconocido teórico canadiense de la comunicación de la segunda mitad del siglo XX caracterizó los grandes momentos históricos en la evolución de la comunicación del ser humano. Las premisas fundamentales de esta taxonomía quedaban resumidas en la conocida sentencia: el medio es el mensaje. $Y$ dado que, de esta forma, se pone en duda cuál es el estímulo y cuál es la reacción al establecerse un enlace comunicacional, la incertidumbre llega hasta el extremo de cuestionarse cuál es el momento en que se está produciendo -de facto- el mensaje mismo. $Y$ pese a todas estas paradojas, lo que resulta más desconcertante aún es dónde se encuentra el mensaje en el momento de establecerse el enlace comunicacional.

Ante tal circunstancia, si las formas y circunstancias en las que se está dando la comunicación transforman, consecuentemente, las formas y circunstancias de las relaciones interpersonales en la sociedad, es lícito imaginar, como señalan Bernete (2014) y Marqués (2012) entre otros muchos, que las TIC digitales están transformando, también, las formas y circunstancias en que se produce la transmisión de la información dentro del aula.

Por ello, antes que buscar la estrategia más expedita para implementar y optimizar el uso de las TIC en el aula, las preguntas fundamentales que deben formularse los docentes y las instituciones educativas, hoy en día, deberían ser: ¿cómo están afectando las TIC digitales al proceso de enseñanza-aprendizaje? y ¿cuán efectiva y deseable es esta transformación?

\section{Marco teórico}

A partir de la segunda mitad del siglo XX, las innovaciones que la sociedad han generado para facilitar las interrelaciones docente-discente, tal y como señalan, McLuhan y Fiore (2009) han estado marcadas, precisamente, por cambios profundos que se han ido sucediendo en los sistemas de comunicación. No obstante, el problema radica en que la inexorabilidad hace que el debate de su implementación en el aula sea visto, preponderantemente, desde su necesaria adecuación y no desde su adecuada necesidad.

Cabe destacar que esas transformaciones debidas a la irrupción de los TIC digitales son ya palpables, conforme hemos mencionado anteriormente y, de acuerdo con Bernete (2010) y Marqués (2012), el fenómeno está siendo ampliamente debatido.

Por otra parte, el tema del uso de las TIC en la sociedad viene siendo, incluso, uno de los más relevantes del Foro Económico Mundial. Esta fundación con sede en Ginebra, Suiza, en la edición 2015 (celebrada en Cancún, México) emitió un claro mensaje en el sentido de emplear las TIC digitales, entre otros factores, para reducir la desigualdad en la región. Sin embargo, la declaratoria nunca puntualizó en cómo se logra este cometido a través de dicha estrategia. 
En este mismo sentido, y casi, con las mismas palabras y elusiones, tal como señala Bernete (2014), se pronunció el Banco Interamericano de Desarrollo al señalar que: "Las TICs tienen un gran potencial para apoyar el desarrollo de mejores aprendizajes y son un factor clave para cerrar las brechas, integrando lo diverso y dando acceso a la información y al conocimiento". Esto nos lleva a plantearnos de nuevo ¿sobre qué bases se apoyan estas aseveraciones?

Al respecto, la reflexión académica sobre el uso de las TIC en el aula se sigue circunscribiendo, en gran medida, a esta forma de enfocar el tema. La incorporación de las tecnologías de la información y la comunicación en las aulas de los centros educativos es considerada por Paucar, Morales y Altamirano (2017) como una medida necesaria y urgente orientada a una mejora significativa en el proceso de aprendizaje y enseñanza.

También, conviene puntualizar que se excluyen del presente análisis los planteamientos que, como el de Marquès (2013), limitan sus posiciones a la necesidad de desarrollar un adecuado adiestramiento operativo de las TIC digitales, sin considerar otros aspectos como su pertinencia y relevancia. Este mismo autor en efecto, habla de un proceso de alfabetización digital al que equipara al de enseñar a leer y a escribir; es decir, el manejo operativo de estas herramientas resulta un medio didáctico más, necesario para acometer los procesos de enseñanza y aprendizaje.

Retomando el objeto de análisis que aquí se plantea, la justificación del uso de las TIC en el aula como herramienta didáctica en sí misma, se fundamenta, en gran medida, desde planteamientos como que éstas "abren una nueva forma de acceder a la información y constituyen un gran puente de comunicación para el contacto y el aprendizaje", Tello y Cascales (2015, p. 355). Con éste un argumento de base, los autores infieren que su uso "permite mejorar la calidad educativa, fomentando la Justicia Social". Así pues, la cuestión sobre cómo se produce este fenómeno es eludida también en este estudio.

Este posicionamiento de partida lleva a Tello y Cascales (2015, pp. 360-361), incluso, a denostar cualquier otro posible enfoque y señalar que "toda la alfabetización digital universal, con sus diversas metodologías de utilización en el aula debe ser justificada mucho más desde el punto de vista de la inclusión social que desde la perspectiva pedagógica". Además, sostienen que la innovación por si sola no es la única clave para mejorar la calidad de la educación, ni conlleva, inexorablemente, a impulsar el aprendizaje y que queda aún por responder cuál es el paradigma pedagógico al que estos estudios buscan dar respuesta.

Bernete (2010) señala que el hecho de que puedan constatarse muchas de las ventajas de utilizar las TIC y que su mera utilización no supone que ello sea siempre igualmente provechoso para todos; y ni siquiera implica, por sí misma, una mejor o peor calidad del aprendizaje.

El determinismo implícito en tales posicionamientos elude un debate más profundo y si cabe aún más urgente, sobre los aspectos deontológicos que se están quedando abiertos, tras la irrupción de las TIC en la sociedad y, consecuentemente, en las estrategias desplegadas en el aula. Siguiendo a Díaz Barriga (2008, p. 1):

Las TIC aplicadas en educación no garantizan por sí mismas ni la inclusión ni la equidad social, tampoco la calidad o la innovación. Por lo que, en muchas ocasiones, la tecnología solo reproduce los modelos de enseñanza tradicionales; por lo que es necesario e imprescindible crear nuevas teorías de diseño educativo con el uso de tecnología que vayan de acuerdo con las necesidades actuales, a fin de lograr un conocimiento útil para la resolución de problemas relevantes y con sentido social. 
En definitiva, la educación debe desarrollar estrategias de integración de nueos métodos ligados a las nuevas tecnologías, de modo que las generaciones actuales y futiuras adquieran competencias digitales que puedan desarrollar en su vida laboral.

Una vez presentado el marco teórico pasamos a presentar cual ha sido la metodología seguida en este estudio.

\section{Metodología}

El presente estudio ha seguido un proceso metodológico de tipo cuantitativo, se ha optado por un estudio tipo encuesta a través del diseño e implementación de un cuestionario, al ser esta técnica la más adecuada si se tiene por objetivo conocer las percepciones de los protagonistas de los procesos educativos (Serrano Pastor 2008). Con los resultados obtenidos se pretende mejorar la enseñanza en el futuro profesorado del Máster de Formación de Profesorado.

\section{Objetivos}

Se toma como punto de partida el siguiente objetivo general:

Analizar el papel de las nuevas tecnologías en el proceso de enseñanza- aprendizaje usadas por profesores de la Facultad de Educación de la Universidad Autonóma de Madrid, que imparten clases dirigidas a futuros profesores de Educación Secundaria Obligatoria en el Máster de Formación de Profesorado.

En consecuencia, se propone el siguiente objetivo específico:

Contrastar las competencias en la formación y el uso que hacen los docentes de las Tic como estrategias de innovación educativa en la Universidad.

\section{Participantes}

La muestra del estudio ha estado compuesta por 60 docentes de la Universidad Autónoma de Madrid que desempeñan su labor como profesores en Máster de Formación de Profesorado tendiendo a las características socio-descriptivas, la mayoría de estos docentes eran 34 profesores, de entre ellas mujeres, 66\%; frente a la población masculina que representó el $34 \%$ del total de la muestra, tal y como queda reflejado en la tabla 1 . En la tabla 2 se presenta la edad de los docentes partcipantes en el estudio cuya edad media es de 31 años.

Gráfico 1.

Género de los docentes participantes en el estudio

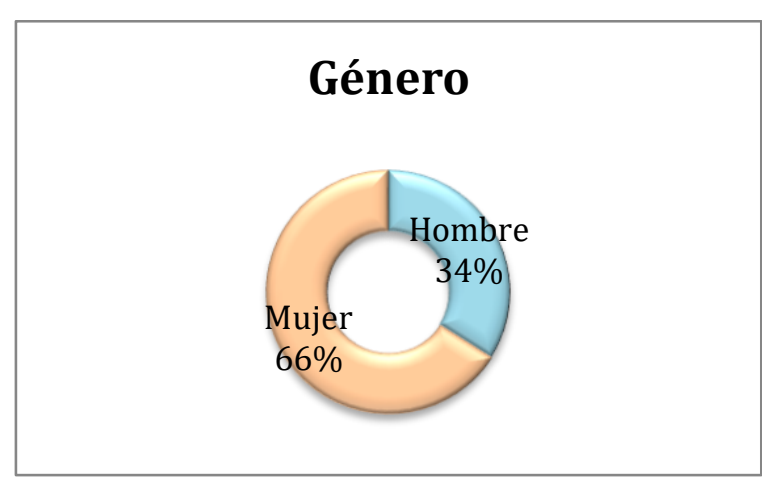


Tabla 2.

Edad de los docentes participantes en el estudio

\begin{tabular}{lllll}
\hline Edad & Frecuencia & $\%$ & $\%$ válido & \% acumulado \\
\hline $21-25$ & 7 & 7 & 7,0 & 9,0 \\
\hline $26-30$ & 36 & 36,0 & 36,0 & 45,0 \\
\hline $31-35$ & 22 & 22.0 & 22.0 & 67,0 \\
\hline $36-40$ & 14 & 14,0 & 14,0 & 81,0 \\
\hline $41-50$ & 10 & 10,0 & 10,0 & 91,0 \\
\hline 51 o más & 9 & 9,0 & 9,0 & 100,0 \\
\hline Total & 100 & 100,0 & 100,0 & \\
\hline
\end{tabular}

\section{Diseño e instrumento de recogida de información}

Con la pretensión de obtener información de las variables de un modo sistemático y ordenado, que intervienen en nuestra investigación, hemos optado, como elemento de carácter cuantitativo, por una de las técnicas más utilizadas en la investigación educativa: el cuestionario ad hoc (Fox, 1981; Kerlinger, 1981; Kemmis y McTaggart, 1988; Cohen y Manion, 1990). Destacando por una de las ventajas que permite la utilización de datos numéricos, con una visión rápida y global, que permite conocer lo que hacen, opinan o piensan los encuestados (Binda y Balbastre, 2013; López-Barajas, 2015).

El cuestionario utilizado ha sido un diseño estructurado en dos bloques del modo que se detalla a continuación:

El primer bloque recoge datos de tipo sociodemográfico y un segundo compuesto por 13 ítems formado por las dimensiones: formación, años de experiencia de los docentes, conocimiento y uso de las TIC en las aulas y uso de ellas en las aulas, cuyas respuestas debían de ser valoradas de acuerdo a una escala tipo Likert (Llauradó, 2014), valorables en un rango de 1 a 5 , en correspondencia con las siguientes categorías de respuesta (1: Nunca; 2: Pocas veces; 3: Algunas veces; 4: Frecuentemente; 5: Mucho o Siempre).

La validación del cuestionario fue realizada a través de diez expertos de otras universidades siendo estos profesores universitarios con formación en contenidos de instrumentos. La fiabilidad del cuestionario fue muy alta, obteniendo un Alfa de Cronbach de 0,95 en el cuestionario para valorar las dimensiones y conseguir los objetivos planteados en este estudio.

\section{Procedimiento}

Los cuestionarios se facilitaron a cada profesor en mano y a través del email de la universidad, se recogieron por parte de los investigadores y se desarrolló la base de datos correspondiente a las preguntas.

Junto con el cuestionario, se envió una carta de presentación de la investigación. Para la recogida de los datos, los docentes contestaron el cuestionario a través de la aplicación Google Forms, y se desarrolló la base de datos en el programa informático SPSS correspondiente a las preguntas que nos ha permitido proceder al análisis de los resultados del estudio. 


\section{Plan de análisis de la información}

Para efectos del presente artículo, se presentan los resultados del núcleo centrado en el análisis estadístico descriptivo de contabilización de frecuencias que han sido traducidas en términos de porcentajes para su difusión en este artículo.

\section{Resultados}

Se presentan a continuación los resultados, que ponen en evidencia que el $42,25 \%$ de los docentes participantes en este estudio conocen las necesidades e inquietudes actuales del alumnado. También son conscientes los participantes de la necesidad de realizar clases dinámicas dentro del proceso de enseñanza-aprendizaje, con relación no solo al conocimiento sobre proceso de Enseñanza-Aprendizaje, si no también en el uso de herramientas tecnológicas en aula y cómo ha cambiado la enseñanza en los últimos tiempos. No obstante, el $73 \%$ de los encuestados evidencia que el profesorado no discrimina suficientemente, según los modelos pedagógicos actuales, los conceptos de enseñanzaaprendizaje. Tampoco los roles que le corresponderían desarrollar tanto al educador como al estudiante en los procesos que se desarrollan en el aula. El estudio evidencia que esta carencia lleva a seleccionar modelos de evaluación no siempre conformes a los objetivos y estrategias pedagógicas planteadas.

Otro de los resultados tal y como queda reflejado en la tabla 3, es que internet en el aula, es usado por el $63 \%$ del profesorado como herramienta didáctica con el fin de dinamizar estas, destacar que el $37 \%$ del profesorado no lo utiliza nunca.

Tabla 3.

El uso de Internet en el profesorado.

\begin{tabular}{lllll}
\hline & Frecuencia & $\%$ & $\%$ válido & \% acumulado \\
\hline 63 & 79 & 63,0 & 63,0 & 63,0 \\
\hline No & 37 & 37,0 & 37,0 & 100,0 \\
\hline Total & 100 & 100 & 100 & \\
\hline
\end{tabular}

Otro de los hallazgos del estudio está relacionado con el número de años de experiencia de los docentes en la universidad y el uso de las Tic (Tabla 4), así a menor número de años de experiencia docente más uso hacen de las Tic. Los profesores con 4,5 años de experiencia en la Universidad es el que mayor uso hace de las nuevas tecnologías en su práctica docente (79\% del profesorado). Sin embargo son los docentes que cuentan con 21 a 30 años de experiencia los que sólo recurren las tecnologías en menos ocasiones ( $2 \%$ de los casos).

Los resultados mostraron que en todas las aulas en las que los docentes habían implementado las nuevas tecnologías, y se hacía uso frecuente de ellas, se traducía en una notable percepción personal positiva frente a la innovación y a las experiencias nuevas por parte de los docentes en áquellos en un $63 \%$. Se observa además, que para su actividad docente establecen una visión más abierta frente a la innovación, y se da una mayor flexibilidad al afrontar las necesidades propias de los proyectos que involucran nuevas tecnologías. 
Tabla 4.

Años de experiencia en la Universidad y uso de las TIC

\begin{tabular}{lllll}
\hline Años de experiencia & Frecuencia & $\%$ & $\%$ válido & \% acumulado \\
\hline $4-5$ & 79 & 79,0 & 79,0 & 79,0 \\
\hline $6-10$ & 19 & 19,0 & 19,0 & 98,0 \\
\hline $21-30$ & 2 & 2 & 2 & 100,0 \\
\hline Total & 100 & 100 & 100 & \\
\hline
\end{tabular}

Tabla 5.

Implementación de las nuevas tecnologías como estrategia didáctica en el aula

\begin{tabular}{lllll}
\hline Uso TIC & Frecuencia & $\%$ & \% válido & \% acumulado \\
\hline $\mathrm{Si}$ & 63 & 63,0 & 63,0 & 63,0 \\
\hline No & 37 & 37,0 & 37,0 & 37,0 \\
\hline Total & 100 & 100 & 100 & \\
\hline
\end{tabular}

Por el contrario, el $37 \%$ de los docentes señaló que, para no perder el respeto de los alumnos, seguían manteniendo estrategias didácticas propias del método tradicional de enseñanza, bien sea porque no conocen técnicas alternativas para aplicar en el aula o por simple elección personal.

La mayoría de los encuestados, el 84\%, indicó conocer y saber manejar las TIC; sin embargo, no se evidenció una coordinación tecno-pedagógica necesaria y suficiente para su uso como herramienta didáctica en el aula.

Respecto al estudio sobre las funciones que pueden desempeñar los docentes en el sistema educativo, cabe señalar su inquietud respecto a su responsabilidad en el tratamiento de las problemáticas del absentismo y del fracaso escolar; en la mediación y resolución de conflictos, así como en otras funciones más propias de la parte gerencial de la docencia, tales como el manejo de la documentación relativa a organización y funcionamiento de los centros; la coordinación profesional y el trabajo en red; la participación en las responsabilidades.

Otra variable que se analizó ha sido la relacionada con los intereses que los alumnos muestran en relación a las nuevas tecnologías en los últimos tiempos. El 72,45\% de los participantes en el estudio señaló la importancias de una enseñanza enfocada al futuro a través del uso de las nuevas tecnologías, que le faciliten su acceso a la vida laboral; mientras que el $27,55 \%$ comentó que aunque los intereses han cambiado no lo consideran un factor tan relevante.

Otra cuestión que nos planteamos fue conocer el uso de internet para las presentaciones en las aulas y los resultados fueron los siguientes:

- Nunca utilizan internet en las aulas en un $7 \%$ de los casos.

- Un $15 \%$ solamente en determinadas ocasiones. 
- Un $41 \%$ frecuentemente y un $37 \%$ que lo usa muy frecuentemente.

Tabla 6.

Uso de las TIC para la presentaciones en el aula.

\begin{tabular}{|c|c|c|c|c|}
\hline $\begin{array}{l}\text { Presentaciones aula } \\
\text { usando TIC }\end{array}$ & Frecuencia & $\%$ & \% válido & $\%$ acumulado \\
\hline Nunca & 7 & 7,0 & 7,0 & 7,0 \\
\hline Algunas veces & 15 & 15,0 & 15,0 & 22,0 \\
\hline Frecuentemente & 2 & 2 & 2 & 63,0 \\
\hline Muy frecuentemente & 37 & 37,0 & 37,0 & 100,0 \\
\hline Total & 100 & 100 & 100 & \\
\hline
\end{tabular}

Tabla 7.

Frecuencia del uso de internet por los profesores en su labor educativa

\begin{tabular}{lllll}
\hline $\begin{array}{l}\text { Frecuencia del uso de } \\
\text { internet en aula }\end{array}$ & Frecuencia & $\%$ & \% válido & \% acumulado \\
\hline Nunca & 43 & 43,0 & 43,0 & 43,0 \\
\hline Algunas veces & 15 & 15,0 & 15,0 & 58,0 \\
\hline Frecuentemente & 37 & 37,0 & 37,0 & 95,0 \\
\hline Muy frecuentemente & 5 & 5,0 & 5,0 & 100,0 \\
\hline Total & 100 & 100,0 & 100 & \\
\hline
\end{tabular}

Cabe destacar que las variables diseñadas para el estudio demostraron que las relaciones obtenidas en la solución factorial son coherentes y lógicamente predecibles. La información recabada en el estudio sobre perfiles de competencias, tanto pedagógicas como tecnológicas, coincide con la percepción que se aprecia en la literatura (Area, 2005; Cabero, 2007; Cabero, 2014; Ortega-Navas, 2010; Cabero-Almenara et al., 2018, entre otros). Tanto el análisis de la literatura como las conclusiones del estudio evidencias una carencia significativa en el manejo y comprensión de conceptos y roles propio del proceso pedagógico de enseñanza-aprendizaje.

Otra de las cuestiones que analizamos ha sido la frecuencia con que el profesorado realizaba tareas de actualización y perfeccionamiento en el uso de las TIC. Para ello nos centramos en la frecuencia con la que los profesores realizaban cursos online y comprobamos que el $46 \%$ de los profesores realiza 2 cursos online al año para actualizarse frente el $21 \%$ afirma que realiza más de 4 cursos al año y el $3 \%$ de los encuestados señala que no realiza ningún curso online al año. 
Tabla 8.

Frecuencia de realización cursos online anualmente

\begin{tabular}{lllll}
\hline Cursos online & Frecuencia & $\%$ & \% válido & \% acumulado \\
\hline Ninguno & 3 & 3,0 & 3,0 & 3,0 \\
\hline 1 & 3 & 3,0 & 3,0 & 6,0 \\
\hline 2 & 46 & 46,0 & 46,0 & 52,0 \\
\hline 3 & 27 & 27,0 & 27,0 & 79,0 \\
\hline 4 o más & 21 & 21,0 & 21,0 & 100,0 \\
\hline Total & 100 & 100,0 & 100 & \\
\hline
\end{tabular}

\section{Discusión y conclusiones}

Cada época ha tenido sus propios paradigmas y enfoques educativos. Y cada uno de éstos ha sido concebido de conformidad a los conceptos y a los instrumentos metodológicos y tecnológicos disponibles.

Las nuevas tecnologías, introducen en el aula diferentes formas de enseñanza formados por una metodología que se pueden compaginar con la enseñanza tradicional. Por lo tanto, la enseñanza de las TIC invita a los discentes a explorar la información.

Cabe destacar que, existe una diferencia esencial entre "información" y “conocimiento". El conocimiento conlleva el poder para crear nuevos significados y estructuras. Estos nuevos significados se materializan como nuevas ideas y estrategias, en escenarios que pueden ser muy diferentes de aquel en el que se originó el conocimiento de base.

En este contexto según Boyd y Ellison (2007) son un medio que permite a los individuos construir un perfil público o semipúblico dentro de un sistema delimitado, articular una lista de otros usuarios con los que comparten una conexión, y ver y recorrer la lista de conexiones de otros miembros del sistema. De manera que las posibilidades de las nuevas herramientas digitales de la comunicación y de la información se presentan muy prometedoras, en la educación, para enriquecer el conocimiento y la enseñanzaaprendizaje.

Ferreira (2014) señala que el uso de las TIC cambia profundamente el impacto de éstas en los procesos sociales y en la relación pedagógica, asumiendo diversas modalidades de autonomía en la formación del alumnado, así como en las formas de relación con las normas y la autoridad como un uso diferenciado de las TIC en los jóvenes.

Los estudiantes no demandan de sus profesores romper la relación asimétrica, sino resignificarla, reconstruirla en una interacción siempre contingente (Zamora y Zerón, 2010), es decir, que encarnen una verdadera autoridad; y señalan sus rasgos: el conocimiento especializado de su materia, su adecuada transmisión, unas reglas claras que se hagan cumplir, un compromiso de respeto recíproco con sus alumnos, el cuidado y la protección mediante vínculos cercanos y personales, saber crear un clima de confianza que guíe y promueva la autonomía del alumno (Pierella, 2011, 2014; Tallone, 2011; Gutiérrez-Cabello, 2015). 
Los profesores señalan que si los alumnos muestran actitudes positivas ante las Tic y las usan más frecuentemente dentro del aula, éstos estarán más preparados para sus futuros trabajos a nivel académico y adquirirán más destrezas importantes en su futura vida profesional Bullón, et al. (2008). Además, que la innovación educativa contribuirá a una mejor formación académica del alumno no solo a nivel académico, si no también cultural y social.

La discusión sobre la irrupción de las TIC en el aula se está produciendo, primordialmente, a nivel didáctico y no tanto a nivel pedagógico; eludiéndose con ello cuestiones fundamentales que no han sido resueltas y aún deben ser abordadas con mayor profundidad para el proceso de enseñanza -aprendizaje.

Siguiendo a Cabrero (2014) se indica que el profesor necesita una capacitación por la interacción de tres grandes componentes: disciplinar, pedagógico y tecnológico. En efecto, los esfuerzos que acometan tanto los centros educativos, a título institucional, como los profesores, de forma particular, para lograr la adecuación tecno-pedagógica en el aula, debe trascender tanto la simple dotación de los medios tecnológicos necesarios, como la capacitación meramente instrumental del profesorado.

La justificación sobre la conveniencia social del uso de estas nuevas herramientas, sin dejar de ser un factor significativo, no debe excluir el debate epistemológico. De hecho, la omisión, sea intencional o displicente, de la reflexión epistemológica del uso de TIC en el aula, por parte de docentes e instituciones, conlleva, inexorablemente, a la perpetuación acrítica de modelos pedagógicos; que pueden resultar muy limitados en cuanto a sus objetivos y posibilidades.

Igualmente, es necesario concienciar en una visión crítica de las TIC por parte del profesorado, lo que supone cambiar su rol, así como tener en cuenta las experiencias de la vida extraescolar (Correa et al., 2015, 54); todo ello se traduce en el aula en la responsabilidad de ejercer su papel de orientador experimentado para promover un uso crítico, emancipador, igualitario y justo de las TIC, es decir, más autoridad y no menos, si bien no de tipo disciplinario, sino demostrativo.

Sin entrar a valorar la idoneidad de alguno de los modelos pedagógicos sobre los otros dos a los que ha hecho referencia este estudio basado en los planteamientos de Kaplún (1985), es imprescindible ahondar en los fundamentos epistemológicos del proceso de enseñanzaaprendizaje; aunque sólo sea asumido con el propósito de seleccionar el esquema evaluativo con las estrategias didácticas utilizadas en el aula.

Por otra parte, las TICs cambian el modo de aprendizaje el tipo de interacción y la filosofía de trabajo (Colmenares y Barroso, 2014) de manera que la utilización de las TIC es ampliamente reconocida y que el uso de TIC en el aula permite desarrollar estrategias didácticas más creativas y participativas para el alumno, que constituyen potencialmente una excelente herramienta didáctica. En consecuencia los mejores resultados sólo se alcanzan en la medida en que el docente tenga claro el modelo pedagógico que quiere impulsar y, por tanto, maneje con soltura los conceptos y roles correspondiente.

Por ultimo, no queremos finalizar sin señalar que resulta particularmente pertinente dada la abundancia de información, a la vez indiscriminada y sin contrastar, que, hoy más que nunca se insista en la búsqueda de estrategias pedagógicas que contribuyan a la mejora en la accesibilidad, desarrollo, innovación y actualización de las competencias digitales en un mundo cada vez más digitalizado. Además, la discusión sobre la irrupción de las TIC en el aula se está produciendo, primordialmente, a nivel didáctico y no tanto a nivel pedagógico; eludiéndose con ello cuestiones fundamentales que no han sido resueltas y aún deben ser abordadas con mayor profundidad para el proceso de enseñanza-aprendizaje. Las nuevas 
tecnologías juegan un papel importante como estrategia innovadora en el proceso de enseñanza- aprendizaje y es un reto que deberíamos plantearnos en el proceso educativo actual.

\section{Referencias}

Area, M. (2005). Tecnologías de la información y la comunicación en el sistema escolar. Una revisión de las líneas de investigación. Revista Electrónica de Investigación y Evaluación Educativa, 11(1), 3-25.

Bruner, J. (2013). La educación puerta de la cultura. Madrid: Antonio Machado.

Bernete, F. (2010). Usos de las TIC. Relaciones sociales y cambios en la socialización de las y los jóvenes. Revista de Estudios de juventud, 88, 97-114.

Bernete, F. (2014). Cambios en la educación asociados a nuevas plataformas comunicativas, en J.F. Duran Medina (Coord.). La era de las TIC en la nueva docencia (pp. 71-84). Madrid: McGraw Hill.

Binda, N.U.y Balbastre,F. (2013). Investigación cuantitativa e investigación cualitativa: buscando las ventajas de las diferentes metodologías de investigación. Pedagogía social. Revista interuniversitaria, 31 (2), 179-187.

Boyd, D. y Ellison, N. (2007). Social Network sites: Definition, History, and Scholarship. Journal of Computer-Mediated Communication, 13 (1). http://jcmc.indiana.edu/vol13/issue1/boyd.ellison.html (Consulta:02/2009).

Bullón, P. et al. (2008). Alfabetización digital de los estudiantes de la Facultad de Odontología de la Universidad de Sevilla. Sevilla: Grupo de Investigación Didáctica.

Cabero, J. (2007). Tecnología Educativa: Diseño y utilización de medios en la enseñanza. Barcelona: Paidós.

Cabero, J. (2014). Formación del profesorado universitario en TIC. Aplicación del método Delphi para la selección de los contenidos formativos. Educación XX1, 17 (1), 111-132. doi: 10.5944/educxx1.17.1.10707

Cabero-Almenara, J. et al. (2018). La aceptación de la Tecnología de la Formación Virtual y su relación con la capacitación docente en docencia virtual. EDMETIC. Revista de Educación Mediática y TIC, 7(1), 225-241. Recuperado de: https://doi.org/10.21071/edmetic.v7i1.10028

Cohen, L., y Manion, L. (1990). Métodos de Investigación educativa. Madrid: La Muralla.

Colmenares, L. y Barroso, J. (2014). Tipos de aprendizajes emergentes bajo la influencia de la Web 2.0. Revista Internacional de Investigación e Innovación educativa. IJERI, 1, 99108.

Correa, J.M., et al. (2015). Formación del Profesorado, Tecnología Educativa e Identidad Docente Digital. RELATEC, Revista Latinoamericana de Tecnología Educativa, 14(1), 4556.

Díaz Barriga, F. (2008). Educación y Nuevas tecnologías de la Información y la comunicación: ¿Hacia un paradigma educativo innovador? Sinéctica. Revista Electrónica de Educación, 30, 1-15. 
Ferreira, N. (2014). La autonomía, autoridad y confianza en un momento de nuevas TIC: distintas actitudes y prácticas de los estudiantes de secundaria. Publicação de Faculdade de Letras da Universidade do Porto.

Fox, D. (1981). El proceso de investigación en educación. Pamplona: Eunsa.

Gutiérrez-Cabello, A. (2015). Entre la autoridad y el autoritarismo: análisis del papel de las tecnologías digitales en la relación pedagógica. REIRE. Revista d'Innovació i Recerca en Educació, 8(2), 357-367. Recuperado de: https://doi.org/110.1344/reire2015.8.28225

Kaplún, M. (1985). El comunicador popular. Quito: CIESPAL.

Kemmis, S. y Mctaggart, R. (1988). The action Research planner. Geelong: Deakin University Press.

Kerlinger, F. N. (1981). Enfoque Conceptual de la Investigación del Comportamiento. México: Editorial Interamericana.

Llauradó, O. (2014) La escala de Likert: qué es y cómo utilizarla. Recuperado de:. https://www.netquest.com/blog/es/la-escala-de-likert-que-es-y-como-utilizarla

López-Barajas Zayas, E. (2015). Introducción a la metodología científica:(Siete piezas fáciles). Universidad Internacional de La Rioja.

Marquès, P.R. (2012). Modelos didácticos de aprendizaje en grupo y aprendizaje colaborativo con TIC. Comunicación y pedagogía. Nuevas tecnologías y recursos didácticos, 261-262, 26-29.

Marquès, P.R. (2013). Impacto de las TIC en la educación: Funciones y limitaciones. 3 C TIC: cuadernos de desarrollo aplicados a las TIC, 2 (1), 1-15. (Ejemplar dedicado a: 3 C TIC Edición $\mathrm{n}^{\circ} 3$ )

McLuhan, M. y Fiore, Q. (2009). El medio es el masaje. Un inventario de efectos. The Medium is the Massage. Random House, 2000.

Miralles-Martínez, P., Gómez-Carrasco, C. J., Arias-González, V. B. y Fontal-Merillas, O. (2019). Recursos digitales y metodología didáctica en la formación inicial de docentes de Historia. Digital resources and didactic methodology in the initial training of History teachers. Comunicar, XVII(61), 45-56. https://doi.org/10.3916/C612019-04

Ortega-Navas, M.C. (2010). Las nuevas tecnologías como instrumentos innovadores de la educación a lo largo de la vida. Revista Española de Pedagogía, 69(3), 323-338.

Paucar, L. Morales, J. y Altamirano, S.H (2017). Dirección y gestión estratégica de las TICS. Revista Dominio de las Ciencias, 3(4), 1150-1160. Recuperado de: https://doi.org/10.23857/pocaip

Pellizzon, M. N. (2012). El uso del blog en la enseñanza a adolescentes como herramienta alternativa complementaria en la modalidad presencial. Rumbos tecnológicos, 5, 2745.

Pierella, M.P. (2011). Una experiencia de formación cara a cara. Los estudiantes de Física y sus visiones acerca de la autoridad. IICE. Revista del Instituto de Investigaciones en Ciencias de la Educación, 30, 139-153.

Pierella, M.P. (2014). La autoridad en la universidad. Vínculos y experiencias entre estudiantes, profesores y saberes. Buenos Aires: Paidós. 
Serrano Pastor, F.J. (2008). El cuestionario en la investigación en ciencias sociales. Seminario de la Asociación Extremeña de Ciencias Matemáticas. Badajoz: Ventura Reyes Prosper.

Tallone, A.E. (2011). Las transformaciones de la autoridad docente, en busca de una nueva legitimidad. Revista de Educación, número extraordinario, 115-135.

Tello, I. y Cascales, A. (2015). Las TIC y las necesidades específicas de apoyo educativo: análisis de las competencias TIC en los docentes. RIED. Revista Iberoamericana de Educación a Distancia, 18 (2), 355-383.

Zamora, G. y Zerón A.M. (2010). Caracterización y sentido actual de la autoridad pedagógica en escuelas chilenas de sectores de pobreza. Revista Española de Pedagogía, 68(245), 99-116. 